\title{
ON THE ASYMPTOTIC BEHAVIOR OF THE SECOND MOMENT OF THE FOURIER TRANSFORM OF A RANDOM MEASURE
}

\author{
MANUEL L. ESQUÍVEL
}

Received 21 October 2002

\begin{abstract}
The behavior at infinity of the Fourier transform of the random measures that appear in the theory of multiplicative chaos of Mandelbrot, Peyrière, and Kahane is an area quite unexplored. For context and further reference, we first present an overview of this theory and then the result, which is the main objective of this work, generalizing a result previously announced by Kahane. We establish an estimate for the asymptotic behavior of the second moment of the Fourier transform of the limit random measure in the theory of multiplicative chaos. After looking at the behavior at infinity of the Fourier transform of some remarkable functions and measures, we prove a formula essentially due to Frostman, involving the Riesz kernels.
\end{abstract}

2000 Mathematics Subject Classification: 42B10, 60G57.

1. Introduction. The problem considered in the second section of this work admits a general formulation that can be stated as follows. A random measure is defined in the sense of a random object (see [10, page 9]) by the action of a random operator on a usual Borel measure in such a way that its Fourier transform is almost surely a uniformly continuous and bounded function. A natural conjecture to be made is that the almost sure behavior, at infinity, of the Fourier transform of the random measure is somehow related to the behavior at infinity of the Fourier transform of the Borel measure used to build this random measure. A technique that has given good results in problems such as the one presented here goes as follows (see [9, pages 253-255, 265-267]). One first gets good estimates on the behavior of the moments of the random functions and then, by an accumulation argument, the almost sure behavior is obtained. The study of the asymptotic behavior of the second moment, besides the instrumental usefulness for the technique described, can give an idea of what to expect on the almost sure behavior.

1.1. Multiplicative chaos: an overview. For future reference and for an understandable context to the following, we explain briefly some of the most important ideas of the beautiful theory of multiplicative chaos. The main references of the plainly developed theory are the masterful expositions [10, 11, 12]. The foundation stones of this circle of ideas may be traced to Mandelbrot's work of 1972 [19] criticizing ideas of Kolmogorov's model for turbulence (1962) and proposing a substitute framework by means of a limit lognormal model. In it, instead of having the average energy dissipation over a cube of fixed radius being lognormal, as in Kolmogorov's model, it is an approximate dissipation of energy depending on a continuous parameter that would be lognormal. 
In that way, and as explained by Mandelbrot in 1977 (see [22]), the normalized exponentiation of Gaussian processes could be a more adjusted interpretation of limit lognormal processes, which would in turn be the correct version of the lognormal hypothesis of Kolmogorov. Later on, in 1974, a couple of notes of Mandelbrot (see [20, 21]) were followed by works of Kahane (see [8]) and Peyrière (see [13, 24]) developing some of the characteristic features of the theory. Extensions, refinements, and a refutal of a conjecture of Kahane were provided later by other authors (see [3, 4, 25, 32]).

For a basic start, let $(\Omega, \mathcal{A}, \mathbb{P})$ be a probability space and consider $\left(X_{n}(t)\right)_{n \in \mathbb{N}}$ a sequence of independent Gaussian centered random functions defined over $T$, a locally compact metric space which for us will be the usual Euclidean normed space $\mathbb{R}^{v}$. We may then define the associated lognormal weights

$$
P_{n}(t):=\exp \left(X_{n}(t)-\frac{1}{2} \mathbb{E}\left[X_{n}^{2}(t)\right]\right)
$$

Observing that $\mathbb{E}\left[P_{n}(t, \cdot)\right]=1$ if we define

$$
Q_{n}(t):=P_{1}(t) \cdot P_{2}(t) \cdots P_{n}(t)
$$

then $\left(Q_{n}(t, \omega)\right)_{n \in \mathbb{N}, t \in \mathbb{R}^{v}, \omega \in \Omega}$ is a positive $\mathbb{R}^{v}$-martingale. That means that:

(1) for each $t_{0} \in \mathbb{R}^{v}$ fixed, $\left(Q_{n}\left(t_{0}, \cdot\right)\right)_{n \in \mathbb{N}}$ is a $\mathscr{C}$ martingale where the filtration $\mathscr{C}=$ $\left(C_{n}\right)_{n \in \mathbb{N}}$ is naturally given by

$$
C_{n}=\sigma\left(\left\{X_{m}: m \leq n\right\}\right)
$$

(2) for almost all $\omega_{0} \in \Omega$, we have that $\left(Q_{n}\left(\cdot, \omega_{0}\right)\right)_{n \in \mathbb{N}}$ is a sequence of positive Borel functions on $\mathbb{R}^{v}$.

Take now $\sigma$ a positive Radon measure on $\mathbb{R}^{v}$ (see [23, page 9] or [18, page 75]) and consider the sequence of random measures defined by $\left(Q_{n} \sigma\right)_{n \in \mathbb{N}}$. The following result ensures the existence of the weak limit of this sequence.

THEOREM 1.1 [12, page 12]. Under the condition

$$
r(t):=\mathbb{E}\left[Q_{n}(t, \cdot)\right] \in L^{1}(\sigma),
$$

$\left(Q_{n} \sigma\right)_{n \in \mathbb{N}}$ converges weakly (i.e., over $C_{0}$ the continuous functions on $\mathbb{R}^{v}$ having zero as a limit at infinity) almost surely to a random measure designated by $S_{\sigma}$.

As a consequence of this result, we may define an operator $Q$ on the positive Radon measures on $\mathbb{R}^{v}, M^{+}=M^{+}\left(\mathbb{R}^{v}\right)$, into the space of random measures by

$$
\forall \sigma \in M^{+}, \quad Q \sigma=S_{\sigma}=\lim _{n \rightarrow+\infty} Q_{n} \sigma
$$

This is by definition the multiplicative chaos operator associated with $\left(X_{n}\right)_{n \in \mathbb{N}}$. A basic fundamental fact is that the distribution of the operator $Q$, namely, the joint distribution of $\left(Q \sigma_{1}\left(B_{1}\right), Q \sigma_{2}\left(B_{2}\right), \ldots, Q \sigma_{n}\left(B_{n}\right)\right)$ for all choices of $n, \sigma_{1}, \ldots, \sigma_{n}, B_{1}, \ldots, B_{n}$, 
depends only on

$$
q(s, t):=\sum_{n=1}^{+\infty} p_{n}(t, s) \leq+\infty,
$$

where we suppose that $p_{n}(t, s):=\mathbb{E}\left[X_{n}(t) \cdot X_{n}(s)\right] \geq 0$.

In this paper, we will be particularly interested in the case where, for a certain parameter $u>0$, we have

$$
q(s, t)=u \ln ^{+}\left(\frac{1}{\|t-s\|_{\mathbb{R}^{v}}}\right)+O(1),
$$

which is a natural model for isotropic turbulence. Suppose that $r \in L^{1}(\sigma)$. In general, there are two extreme cases concerning the image of operator $Q$ :

(1) either $Q \sigma \equiv 0$ almost surely in which case we say that $Q$ is degenerate on $\sigma$;

(2) or the martingale $\left(Q_{n} \sigma(B)\right)_{n \in \mathbb{N}}$ converges in $L^{1}(\Omega)$ for each given Borel set $B$. This means that

$$
\mathbb{E}[Q \sigma](B)=r \sigma B=\int_{B} r(t) d \sigma(t)
$$

which we represent by

$$
\mathbb{E} Q \sigma=\mathbb{E}\left[\int d S_{\sigma}\right]=\int r d \sigma
$$

and is usually described by saying that $Q$ is fully acting on $\sigma$ or $Q$ lives on $\sigma$. It is possible to show [12, page 13] that for each $\varphi \in C_{0}$ we have

$$
\mathbb{E}\left[\int \varphi d S_{\sigma}\right]=\int \varphi r d \sigma .
$$

In the case where $q(s, t)$ is given by formula (1.7), every compact having Hausdorff dimension greater than $u / 2$ supports measures such that $Q \sigma \neq 0$.

When dealing with moments, it is particularly useful to consider the $L^{2}$ theory. One may say that $Q$ is strongly nondegenerate on $\sigma$ if for every compact $K$ of $\mathbb{R}^{v}$ we have

$$
\mathbb{E} Q \sigma(K)=\sigma(K)
$$

The $L^{2}$ theory gives some conditions for $Q$ to be strongly nondegenerate on $\sigma$.

THEOREM 1.2 [10, page 133]. The following are equivalent:

(i) $Q$ is strongly nondegenerate in $\sigma$, and moreover $\mathbb{E}[Q \sigma(K)]^{2}<+\infty$;

(ii) $\int_{K} \int_{K} e^{p_{n}(t, s)} d \sigma(t) d \sigma(s)=O(1)$;

(iii) $\int_{K} \int_{K} e^{q(t, s)} d \sigma(t) d \sigma(s)<+\infty$.

Under condition (iii) of Theorem 1.2, if $k(t)$ is a complex bounded function over $\mathbb{R}^{v}$, we have

$$
\mathbb{E}\left[\left|\int_{K} k(t) d S_{\sigma}\right|^{2}\right]=\int_{K} \int_{K} k(t) \overline{k(s)} e^{q(t, s)} d \sigma(t) d \sigma(s) .
$$


For $q(s, t)$ given by formula (1.7), condition (iii) of Theorem 1.2 says that $\sigma$ has finite $u$-energy (see for a definition [23, page 109] or Theorem 3.4). As a consequence (see [12, page 45]), for $u<d$ we have $Q$ lives on $\sigma$ whenever $\sigma$ has finite $u$-energy.

We define the Fourier transform of the random measure $S_{\sigma}$. Under the main hypothesis of Theorem 1.1, namely, $r \in L^{1}(\sigma)$, we have almost surely

$$
\lim _{n \rightarrow+\infty} \int Q_{n} d \sigma=S_{\sigma}(1)<+\infty
$$

as a consequence of formula (1.9). We can then conclude that there is a convergence over the bounded continuous functions on $\mathbb{R}^{v}$ (see [18, page 98]). As a consequence, the definition of the Fourier transform of the random measure $S_{\sigma}$ is straightforward.

DEFINITION 1.3. The Fourier transform $\hat{S}_{\sigma}$, of the random measure $S_{\sigma}$, is by definition the map defined almost surely by

$$
\forall \xi \in \mathbb{R}^{v}, \quad \hat{S}_{\sigma}(\xi)=\lim _{n \rightarrow+\infty} \int_{\mathbb{R}^{v}} \exp (2 \pi i \xi t) Q_{n} \sigma(d t)
$$

As usual (see [14, page 132]), it is easily verified that almost surely $\hat{S}_{\sigma}$ is uniformly continuous and that for a bounded and positive Radon measure $\sigma$ the map $\hat{S}_{\sigma}$ is almost surely bounded.

REMARK 1.4. With additional hypothesis, it can be verified that $S_{\sigma}$ is a random measure in the sense of a measurable map taking its values in a (measurable) space of measures. More precisely, if the operator $Q$ is strongly nondegenerate, then the martingale defined for all $\varphi \in C_{0}\left(\mathbb{R}^{v}\right)$ by $\left(\int \varphi Q_{n} d \sigma\right)_{n \in \mathbb{N}}$ is an $L^{2}$ martingale. In fact, for some constants $c, A$,

$$
\forall n \in \mathbb{N}, \quad \mathbb{E}\left[\left|\int \varphi Q_{n} d \sigma\right|^{2}\right] \leq c \iint \exp \left(q_{n}(t, s)\right) d \sigma(t) d \sigma(s) \leq A<+\infty .
$$

Following [2], we can say that the sequence of random measures $\left(Q_{n} \sigma\right)_{n \in \mathbb{N}}$ converges in quadratic mean (see [2, page 49]). This shows that $S_{\sigma}$ is a random measure when considered as a map defined on a probability space and taking its values in the space of the Radon signed measures, which is a measurable space, when endowed with the Borel $\sigma$ algebra associated with the topology of vague convergence. The random measures associated to the multiplicative chaos are in this way and under some restrictive hypothesis nontrivial examples of random signed measures in the sense of Kallemberg and Oliveira.

2. On the asymptotic behavior. Asymptotic behavior of the Fourier transform of a measure is a classical subject in harmonic analysis (see [15, page 218], [23, page 168], [30, pages 347-351, 360-364]). This subject has received some attention recently. In part, due to the relevance for applications of the $L^{2}$ energy norm, the behavior of second-order moments is particularly interesting (see, e.g., [6, 17, 31, 33]).

The main result furnished by the established theory on the asymptotic behavior of the Fourier transform of the random measure $S_{\sigma}$ is the following. 
THEOREM 2.1 [10, page 135]. If $q(s, t)$ given by formula $(1.7)$ is a bounded $C^{\infty}$ function with compact support and if $\sigma$ has compact support and a $C^{\infty}$ density with respect to the Lebesgue measure, then

$$
\mathbb{E}\left[\left|\hat{S}_{\sigma}(\xi)\right|^{2}\right] \asymp\|\xi\|^{u-v} .
$$

The main purpose of this work is to prove the following extension of this result. It gives the asymptotic behavior of the second moment in the case where $\sigma$ is a positive Radon measure with compact support admitting an $L^{2}$ density.

THEOREM 2.2. If $q(s, t)$ given by formula (1.7) and $\sigma$ is a positive Radon measure with compact support on $\mathbb{R}^{v}$, such that

$$
\int_{\mathbb{R}^{v}}|\hat{\sigma}(x)|^{2} d x<+\infty, \quad \forall \xi \in \mathbb{R}^{v}, \quad \int_{\mathbb{R}^{v}} \frac{|\hat{\sigma}(x)|^{2}}{\|x-\xi\|^{v-u}} d x<+\infty
$$

and such that the operator $Q$ is strongly nondegenerate on $\sigma$, then, for some constants $c$ and $d$,

$$
\mathbb{E}\left[\left|\hat{S}_{\sigma}(\xi)\right|^{2}\right] \leq \frac{1}{\|\xi\|^{v-u}}\left(c+d\|\xi\|^{v} \sup _{\|x-\xi\|<\|\xi\| / 2}|\hat{\sigma}(x)|^{2}\right)
$$

Proof. We use the result in [10] which says that

$$
\mathbb{E}\left[|\hat{S}(\xi)|^{2}\right]=\mathbb{E}\left[\left|\int \exp (2 \pi i t \xi) d S(t)\right|^{2}\right]=\int_{\left(\mathbb{R}^{v}\right)^{2}} \exp (2 \pi i(t-s) \xi) e^{q(t, s)} d \sigma(t) d \sigma(s)
$$

as a consequence of formula (1.12). We will deal first with the special case where $\sigma$ admits a $C^{\infty}$ density $f$ with compact support. Suppose then that $d \sigma(t)=f(t) d t$. By a trivial change of variables and by Fubini's theorem, we get

$$
\begin{aligned}
\int_{\mathbb{R}^{2 v}} \frac{e^{-2 \pi i(t-s) \xi}}{\|t-s\|^{u}} d \sigma(t) d \sigma(s) & =\int_{\mathbb{R}^{2 v}} \frac{e^{-2 \pi i v \xi}}{\|v\|^{u}} f(v+s) f(s) d v d s \\
& =\int_{\mathbb{R}^{v}} \frac{e^{-2 \pi i v \xi}}{\|v\|^{u}}(f * \check{f})(s) d v .
\end{aligned}
$$

The hypotheses on $f$ imply that $(f * \check{f})$ is a $C^{\infty}$ function with compact support strictly positive in a neighborhood of zero. As a consequence of Proposition 3.3, we get for some constant $c$,

$$
\mathbb{E}\left[|\hat{S}(\xi)|^{2}\right] \leq \frac{c}{\|\xi\| \min (u,(v+1) / 2)},
$$

which is a weaker result than the one announced for $u \geq(v-1) / 2$. The general case needs another kind of approach. We apply Theorem 3.4 to the last term in formula (2.4) to get for some constant $d$,

$$
\mathbb{E}\left[|\hat{S}(\xi)|^{2}\right] \leq d \int_{\mathbb{R}^{v}} \frac{|\hat{\sigma}(x)|^{2}}{\|x-\xi\|^{v-u}} d x
$$


Denote by $I$ the following integral:

$$
I=\int_{\mathbb{R}^{n}} \frac{|\hat{\sigma}(x)|^{2}}{\|x-\xi\|^{a}} d x .
$$

In order to obtain the asymptotic behavior of this integral, we consider a point $\xi$, fixed in $\mathbb{R}^{n}$ and the partition of the domain of integration given by

$$
\mathbb{R}^{n}=B\left(0, \frac{\|\xi\|}{2}\right) \cup B(\xi, \alpha) \cup\left\{x \in \mathbb{R}^{n}:\|x\| \geq \frac{\|\xi\|}{2},\|x-\xi\| \geq \alpha\right\}
$$

where $\alpha$ is a parameter that we will deal with, below. Let $I_{1}$ (resp., $I_{2}, I_{3}$ ) be the integral of the function $|\hat{\sigma}(x)|^{2} /\|x-\xi\|^{a}$ over the set on the left (resp., on the middle, on the right) of the partition (2.9). Then, it is clear that

$$
\begin{aligned}
& I_{1} \leq \frac{1}{\|\xi\|^{a}} \int_{\|x\| /\|\xi\|<1 / 2} \frac{|\hat{\sigma}(x)|^{2}}{|1-\|x\| /\|\xi\||^{a}} d x \leq \frac{1}{\|\xi\|^{a}} 2^{a}\|\hat{\sigma}\|_{2}^{2}, \\
& I_{2} \leq \sup _{\|x-\xi\|<\alpha}|\hat{\sigma}(x)|^{2} \int_{\|x\|<\alpha} \frac{d x}{\|x\|^{a}} \leq \alpha^{v-a} \sup _{\|x-\xi\|<\alpha}|\hat{\sigma}(x)|^{2} \int_{\|x\|<\alpha} \frac{d x}{\|x\|^{a}}, \\
& I_{3} \leq \frac{1}{\alpha^{a}} \int_{\|x\|>\|\xi\| / 2}|\hat{\sigma}(x)|^{2} d x \leq \frac{\|\hat{\sigma}\|_{2}^{2}}{\alpha^{a}} .
\end{aligned}
$$

As a consequence, for some constants $c$ and $d$ and choosing $\alpha=\|\xi\| / 2$ in (2.11) and in (2.12) we have that

$$
I \leq \frac{1}{\|\xi\|^{a}}\left(c+d\|\xi\|^{v} \sup _{\|x-\xi\|<\|\xi\| / 2}|\hat{\sigma}(x)|^{2}\right)
$$

as desired.

REMARK 2.3. Let $\sigma$ be the Lebesgue measure concentrated on the unit ball of $\mathbb{R}^{v}$. As a consequence of formula (3.1), we will have that for some constant $c$,

$$
|\hat{\sigma}(x)|^{2} \leq \frac{c}{\|x\|^{v+1}}
$$

and as a consequence,

$$
\mathbb{E}\left[|\hat{S}(\xi)|^{2}\right] \leq \frac{c}{\|\xi\|^{v-u}}
$$

in agreement with the result stated in [10, page 30].

REMARK 2.4. The final conclusion in the statement of Theorem 2.2 clearly depends on the asymptotic behavior of the Fourier transform of the measure $\sigma$. We present next an example (given by [27]) that shows that in general the integral in (2.8) has no rate of decay better than $O(1)$. We will see that the measure under scrutiny has not compact support. As a consequence, a natural question is to find an example such as the one presented but with a measure with compact support. 
Consider a sequence of functions $\left(\varphi_{n}\right)_{n \in \mathbb{N}}$ defined by

$$
\forall n \in \mathbb{N}, \quad \varphi_{n}=\mathbb{\square}_{[-n, 1-n]}+\mathbb{\square}_{[n-1, n]} .
$$

As $\varphi_{n}$ is an even function, its Fourier $\widehat{\varphi_{n}}$ transform is real valued. A quick computation shows that

$$
\forall n \in \mathbb{N}, \quad \widehat{\varphi_{n}}(\xi)=\frac{2 \sin (\pi \xi) \cos ((2 n-1) \pi \xi)}{\pi \xi}
$$

Define now a sequence of functions $\left(\psi_{n}\right)_{n \in \mathbb{N}}$ by

$$
\forall n \in \mathbb{N}, \quad \psi_{n}=\varphi_{n} * \varphi_{n}
$$

A simple but tedious computation shows that $\psi_{n}(x)$ is a linear by pieces continuous function with compact support, simply described as the sum of three tent functions given by

$$
\begin{aligned}
\psi_{n}(x)= & (2 n+x) \rrbracket_{[-2 n,-2 n+1]}(x)+(-x-2 n+2) \rrbracket_{[-2 n+1,-2 n+2]}(x) \\
& +(2 x+2) \rrbracket_{[-1,0]}(x)+(2-2 x) \rrbracket_{[0,1]}(x) \\
& +(x-2 n+2) \rrbracket_{[2 n-2,2 n-1]}(x)+(2 n-x) \rrbracket_{[2 n-1,2 n]}(x) .
\end{aligned}
$$

As $\widehat{\psi}_{n}=\left(\widehat{\varphi_{n}}\right)^{2}$, we have that

$$
\forall n \in \mathbb{N}, \quad \hat{\psi}_{n}(\xi)=\frac{4 \sin ^{2}(\pi \xi) \cos ^{2}((2 n-1) \pi \xi)}{\pi^{2} \xi^{2}} .
$$

This shows that $\psi_{n}$ belongs to $L^{1}(\mathbb{R}) \cap L^{2}(\mathbb{R})$. Take a sequence $\left(a_{n}\right)_{n \in \mathbb{N}^{*}}$ of nonnegative numbers such that $\sum_{n=1}^{+\infty} a_{n}<+\infty$ and define a measure $d \sigma(\xi)=f(\xi) d \xi$ with

$$
\forall \xi \in \mathbb{R}, \quad f(\xi)=\sum_{n=1}^{+\infty} a_{n} \hat{\psi}_{n}(\xi) .
$$

As $\sigma(\mathbb{R})=2 \sum_{n=1}^{+\infty} a_{n}$, the measure $\sigma$ is finite. Moreover, as a consequence of CauchySchwarz inequality, the density of $\sigma$ with respect to the Lebesgue measure is in $L^{2}(\mathbb{R})$. In fact, we have

$$
\begin{aligned}
\left(\int_{\mathbb{R}} f^{2}(\xi) d \xi\right)^{1 / 2} & =\left(\sum_{n, m=1}^{+\infty} a_{n} a_{m} \int_{\mathbb{R}} \hat{\psi}_{n}(\xi) \hat{\psi}_{m}(\xi) d \xi\right)^{1 / 2} \\
& \leq\left\|\psi_{n}\right\|_{2}\left\|\psi_{m}\right\|_{2}\left(\sum_{n=1}^{+\infty} a_{n}^{2}\right)^{1 / 2}\left(\sum_{m=1}^{+\infty} a_{m}^{2}\right)^{1 / 2}<+\infty
\end{aligned}
$$

Observe now that for every $p \in \mathbb{N}$,

$$
|\hat{\sigma}(x)|=\frac{1}{2 \pi} \sum_{n=1}^{+\infty} a_{n} \psi_{n}(x) \geq a_{p} \psi_{p}(x) .
$$


As a consequence, for some constant $c$,

$$
I(2 n-1)=\int_{\mathbb{R}} \frac{|\hat{\sigma}(x)|^{2}}{|x-(2 n-1)|^{a}} d x \geq \int_{2 n-2}^{2 n}|\hat{\sigma}(x)|^{2} \geq c a_{n}^{2} .
$$

Finally, by choosing, for example,

$$
a_{n}= \begin{cases}\frac{1}{k^{2}}, & \text { if } n=2^{2^{k}} \\ 0, & \text { otherwise }\end{cases}
$$

we see that $I$ has no rate of decay better than $O(1)$.

3. Auxiliary results and methodological remarks. In this section, we state some results that were used in the proof of Theorem 2.2. Hereafter, letters $c, d$, and $e$ will denote constants not necessarily the same at every instance.

3.1. Some remarkable Fourier transforms. The asymptotic behavior of the Fourier transform of the indicator function of the unit ball in $\mathbb{R}^{n}$, as given in the following proposition, is well known (see [5] or [28, page 51]).

Proposition 3.1. Let $B=B(0,1)=\left\{x \in \mathbb{R}^{n}:\|x\|<1\right\}$ be the unit ball of $\mathbb{R}^{n}$ and $U=\rrbracket_{B}$ the indicator function of $B$. Then, for some constant $c$,

$$
|\hat{U}(\xi)| \leq \frac{c}{|\xi|^{(n+1) / 2}} .
$$

We get a similar result when the unit ball is replaced by $B(0, \delta)$ and the indicator function appears multiplied by a remarkable locally integrable radial function.

Proposition 3.2. Let $B_{\delta}=B(0, \delta)=\left\{x \in \mathbb{R}^{n}:\|x\|<\delta\right\}$ be a ball of $\mathbb{R}^{n}$, centered in zero with radius $\delta>0$ and, for $0<\alpha<n$, the function defined by

$$
U_{\delta}^{\alpha}(x)=\frac{\rrbracket_{B_{\delta}}(x)}{\|x\|^{\alpha}} .
$$

Then, for some constants denoted always by $c$,

$$
\left|\hat{U}_{\delta}^{\alpha}(\xi)\right| \leq \begin{cases}\frac{c}{\|\xi\|^{n-\alpha}}, & \text { if } \alpha>\frac{n-1}{2}, \\ \frac{c}{\|\xi\|^{(n+1) / 2}}, & \text { if } \alpha \leq \frac{n-1}{2}\end{cases}
$$

As a consequence of the last proposition, we obtain the asymptotic behavior of a Fourier transform that was used in the proof of Theorem 2.2.

Proposition 3.3. Let $g$ be a $C^{\infty}\left(\mathbb{R}^{n}\right)$ function with compact support such that $g$ is strictly positive in a neighborhood of zero. Let $0<\alpha<n$ and define $I_{\alpha, g}$, a Fourier transform, by

$$
I_{\alpha, g}(\xi)=\int_{\mathbb{R}^{n}} \frac{e^{-2 \pi i \xi \cdot x}}{\|x\|^{\alpha}} g(x) d x .
$$


The asymptotic behavior of $I_{\alpha, g}$ is the same as the asymptotic behavior of $\hat{U}_{1}^{\alpha}$, that is, for some constant $c$,

$$
\left|I_{\alpha, g}(\xi)\right| \leq \frac{c}{\|\xi\|^{\min (n-\alpha,(n+1) / 2)}}
$$

The proof follows an argument of localization.

3.2. Some Parseval formulas and tempered distributions. The result in this subsection was used in the proof of the main theorem to express the second moment of the Fourier transform of the random measure $S_{\sigma}$.

For $0<\alpha<n$, let $U^{\alpha}$ denote the locally integrable function, defined by

$$
U^{\alpha}(x)=\frac{1}{\|x\|^{\alpha}} \rrbracket_{\mathbb{R}} * n(x),
$$

where $\mathbb{R}^{* n}=\mathbb{R}^{n}-\{0\}$. This function defines a tempered distribution whose Fourier transform denoted by $\hat{U}^{\alpha}$ but also by $\mathscr{F} U^{\alpha}$ is represented again by a locally integrable function given by

$$
\hat{U}^{\alpha}(\xi)=\frac{c(\alpha)}{\|\xi\|^{n-\alpha}} \mathbb{Q}_{\mathbb{R} * n}(\xi), \quad c(\alpha)=\frac{\Gamma((n-\alpha) / 2)}{\pi^{n / 2-\alpha} \Gamma(\alpha / 2)} .
$$

(See [29, page 117] or [16, pages 52, 278].)

This result essentially given by Frostman is usually formulated for real measures and with no exponential term in formula (3.9) (see [1, page 22]).

THEOREM 3.4. Let $\sigma$ be a positive Radon measure over $\mathbb{R}^{n}$ with compact support and $0<\alpha<n$ such that $E_{\alpha}$, the $\alpha$ energy of $\sigma$, is finite, that is,

$$
E_{\alpha}=\int_{\mathbb{R}^{n}} \int_{\mathbb{R}^{n}} \frac{d \sigma(t) d \sigma(s)}{\|t-s\|^{\alpha}}<+\infty
$$

Then,

$$
\int_{\mathbb{R}^{n}} \int_{\mathbb{R}^{n}} \frac{e^{2 i \pi \xi(t-s)}}{\|t-s\|^{\alpha}} d \sigma(t) d \sigma(s)=c(\alpha) \int_{\mathbb{R}^{n}} \frac{|\hat{\sigma}(x)|^{2}}{\|x-\xi\|^{n-\alpha}} d x,
$$

whenever the integral on the right is finite.

Proof. The formula we have to prove is verified for measures given by $d \sigma(t)=$ $\phi(t) d t$, where $\phi \in \mathscr{Y}, \mathscr{Y}$ being the Schwartz test function space of rapidly decreasing functions. Indeed, for such a measure the integral on the left-hand side of formula (3.9) which we denote by $I$ is written as

$$
I=\int_{\mathbb{R}^{n}}\left(\int_{\mathbb{R}^{n}} \frac{e^{2 i \pi \xi t}}{\|t-s\|^{\alpha}} \phi(t) d t\right) e^{-2 i \pi \xi s} \phi(s) d s=\int_{\mathbb{R}^{n}} \frac{(2 \pi)^{n-\alpha}}{c(n-\alpha)} I_{n-\alpha}(h)(s) \bar{h}(s) d s,
$$


where, for $0<\beta<n$, the $\beta$ Riesz potential of $f$ is given by

$$
I_{\beta}(f)(x)=\frac{c(\beta)}{(2 \pi)^{\beta}} \int_{\mathbb{R}^{n}} \frac{f(y)}{\|x-y\|^{n-\beta}} d y
$$

and $h$ stands for $h(s)=e^{2 i \pi \xi s} \phi(s)$. Now, given $f, g \in \mathscr{S}$, we have that

$$
\int_{\mathbb{R}^{n}} I_{\beta}(f)(x) \bar{g}(x) d x=\int_{\mathbb{R}^{n}} \frac{\hat{f}(x) \overline{\hat{g}}(x)}{(2 \pi\|x\|)^{\beta}} d x,
$$

which is essentially Parseval's formula (see [29, page 117] for all the properties of the notion of Riesz potential used). Observing that $\hat{h}(y)=\hat{\phi}(y-\xi)$ and $\bar{h}=\hat{\phi}(\xi-y)$, we have

$$
I=\frac{1}{c(n-\alpha)} \int_{\mathbb{R}^{n}} \frac{\hat{\phi}(y-\xi) \hat{\phi}(\xi-y)}{\|y\|^{n-\alpha}} d y
$$

which gives the result claimed in the statement of the theorem by a trivial change of variables, noticing that, as $\phi$ is real,

$$
\hat{\phi}(u) \hat{\phi}(-u)=\hat{\phi}(u) \overline{\hat{\phi}}(u)=|\hat{\phi}(u)|^{2},
$$

and that $C(\alpha)=1 / c(n-\alpha)$. Let now $\mu$ be a complex measure with compact support and, for $0<\beta<n$, define $I_{\beta}$, the $\beta$ Riesz potential of $\mu$, by

$$
I_{\beta}(\mu)(x)=\frac{c(\beta)}{(2 \pi)^{\beta}}\left(\mu * U^{n-\alpha}\right) .
$$

This definition makes good sense as we are considering the convolution of $\mu$, a distribution with compact support, with $U^{n-\alpha}$ which is a tempered distribution. Observe that as a consequence of a theorem of Sobolev (see [26, page 181]), $I_{\beta}(\mu)$ is locally in $L^{q}$ for $q<n /(n-\beta)$ and in particular $I_{\beta}(\mu)$ is integrable over any compact of $\mathbb{R}^{n}$. Moreover, the Fourier transform of $I_{\beta}(\mu)$ in the sense of distributions is easily computed (see, e.g., [7, page 21]) to give

$$
\widehat{I_{\beta}(\mu)}=\frac{c(\beta)}{(2 \pi)^{\beta}} \hat{\mu} \cdot \hat{U}^{n-\beta}=\frac{1}{(\pi)^{\beta}} \hat{\mu} \cdot \hat{U}^{n-\beta} .
$$

Considering now $d \mu(t)=e^{2 \pi i \xi \cdot t} d \sigma(t)$, we have that

$$
I=\int_{\mathbb{R}^{n}}\left(\int_{\mathbb{R}^{n}} \frac{d \mu(t)}{\|t-s\|^{\alpha}}\right) d \bar{\mu}(s)=\frac{(2 \pi)^{\alpha}}{c(n-\alpha)} \int_{\mathbb{R}^{n}} I_{n-\alpha}(\mu)(s) d \bar{\mu}(s) .
$$

As $\mu$ has compact support and as a consequence of the hypotheses done on the integral 
on the right-hand side of formula (3.9) we can apply Parseval's formula (as given, e.g., in [14, page 132] or [18, page 121]) to obtain

$$
\left.I=\frac{(2 \pi)^{\alpha}}{c(n-\alpha)} \int_{\mathbb{R}^{n}} \widehat{I_{n-\alpha}(\mu}\right)(x) \hat{\bar{\mu}}(x) d x=c(\alpha) \int_{\mathbb{R}^{n}} \hat{\mu}(x) U^{\alpha}(x) \hat{\bar{\mu}}(-x) d x,
$$

which, after some computations of Fourier transforms and a change of variables, is exactly the desired result.

ACKNOWLEDGMENTS. We hereby express our gratitude to the anonymous referees who suggested ideas for a much needed rewriting of the first version of this paper. This work was done with partial support from the FCT (Fundação para a Ciência e Tecnologia), program POCTI (Portugal/FEDER-EU).

\section{REFERENCES}

[1] L. Carleson, Selected Problems on Exceptional Sets, Van Nostrand Mathematical Studies, vol. 13, D. Van Nostrand, Princeton, 1967.

[2] P. E. De Oliveira, Infinie divisibilité, principes d'invariance et estimation de noyaux de transition en théorie des mesures aléatoires, Ph.D. thesis, University of Lille, Lille, 1991.

[3] A. H. Fan, Une condition suffisante d'existence du moment d'ordre $m$ (entier) du chaos multiplicatif, Ann. Sci. Math. Québec 10 (1986), no. 2, 119-120 (French).

[4] _ Sur les chaos de Lévy stables d'indice $0<\alpha<1$, Ann. Sci. Math. Québec 21 (1997), no. 1, 53-66 (French).

[5] C. Fefferman, The multiplier problem for the ball, Ann. of Math. (2) 94 (1971), 330-336.

[6] C.-A. Guerin and M. Holschneider, On equivalent definitions of the correlation dimension for a probability measure, J. Statist. Phys. 86 (1997), no. 3-4, 707-720.

[7] L. Hörmander, Linear Partial Differential Operators, Die Grundlehren der mathematischen Wissenschaften, vol. 116, Springer-Verlag, Berlin, 1963.

[8] J.-P. Kahane, Sur le modèle de turbulence de Benoît Mandelbrot, C. R. Acad. Sci. Paris Sér. A 278 (1974), 621-623 (French).

[9] __ Some Random Series of Functions, 2nd ed., Cambridge Studies in Advanced Mathematics, vol. 5, Cambridge University Press, Cambridge, 1985.

[10] Sur le chaos multiplicatif, Ann. Sci. Math. Québec 9 (1985), no. 2, 105-150 (French).

[11] Positive martingales and random measures, Chinese Ann. Math. Ser. B 8 (1987), no. 1, 1-12.

[12] _ Random multiplications, random coverings, multiplicative chaos, Analysis at Urbana, Vol. I (Urbana, Ill, 1986/1987), London Math. Soc. Lecture Note Ser., vol. 137, Cambridge University Press, Cambridge, 1989, pp. 196-255.

[13] J.-P. Kahane and J. Peyrière, Sur certaines martingales de Benoît Mandelbrot, Adv. Math. 22 (1976), no. 2, 131-145 (French).

[14] Y. Katznelson, An Introduction to Harmonic Analysis, 2nd ed., Dover Publications, New York, 1976.

[15] V. P. Khavin and N. K. Nikol'skiĭ (eds.), Commutative Harmonic Analysis. IV, Encyclopaedia of Mathematical Sciences, vol. 42, Springer-Verlag, Berlin, 1992.

[16] V.-K. Khoan, Distributions, Analyse de Fourier, Opérateurs aux Derivées Partielles, Vols I, II, Librairie Vuibert, Paris, 1972.

[17] K. A. Makarov, Asymptotic expansions for Fourier transform of singular self-affine measures, J. Math. Anal. Appl. 187 (1994), no. 1, 259-286.

[18] P. Malliavin, Integration and Probability, Graduate Texts in Mathematics, vol. 157, SpringerVerlag, New York, 1995. 
[19] B. Mandelbrot, Possible refinement of the lognormal hypothesis concerning the distribution of energy dissipation in intermittent turbulence, Statistical Models and Turbulence, Lecture Notes in Physics, vol. 12, Springer, New York, 1972, pp. 333-351.

[20] __ Multiplications aléatoires itérées et distributions invariantes par moyenne pondérée aléatoire, C. R. Acad. Sci. Paris Sér. A 278 (1974), 289-292 (French).

[21] _ Multiplications aléatoires itérées et distributions invariantes par moyenne pondérée aléatoire: quelques extensions, C. R. Acad. Sci. Paris Sér. A 278 (1974), 355-358 (French).

[22] _ The Fractal Geometry of Nature, W. H. Freeman and Company, New York, 1983.

[23] P. Mattila, Geometry of Sets and Measures in Euclidean Spaces, Cambridge Studies in Advanced Mathematics, vol. 44, Cambridge University Press, Cambridge, 1995.

[24] J. Peyrière, Turbulence et dimension de Hausdorff, C. R. Acad. Sci. Paris Sér. A 278 (1974), 567-569 (French).

[25] H. Sato and M. Tamashiro, Multiplicative chaos and random translation, Ann. Inst. H. Poincaré Probab. Statist. 30 (1994), no. 2, 245-264.

[26] L. Schwartz, Théorie des Distributions, Publications de l'Institut de Mathématique de l'Université de Strasbourg, no. IX-X, Hermann, Paris, 1966.

[27] G. Sinnamon and G. Zimmerman, personal communication.

[28] C. D. Sogge, Fourier Integrals in Classical Analysis, Cambridge Tracts in Mathematics, vol. 105, Cambridge University Press, Cambridge, 1993.

[29] E. M. Stein, Singular Integrals and Differentiability Properties of Functions, Princeton Mathematical Series, vol. 30, Princeton University Press, New Jersey, 1970.

[30] _ Harmonic Analysis: Real-Variable Methods, Orthogonality, and Oscillatory Integrals, Princeton Mathematical Series, vol. 43, Princeton University Press, New Jersey, 1993.

[31] R. S. Strichartz, Self-similar measures and their Fourier transforms. I, Indiana Univ. Math. J. 39 (1990), no. 3, 797-817.

[32] E. C. Waymire and S. C. Williams, Multiplicative cascades: dimension spectra and dependence, J. Fourier Anal. Appl. (1995), 589-609, Special Issue: Proceedings of the Conference in Honor of Jean-Pierre Kahane.

[33] B. Wu and W. Su, Fourier transformation and singular integrals on self-similar measure, Approx. Theory Appl. (N.S.) 14 (1998), no. 4, 102-114.

Manuel L. Esquível: Departamento de Matemática, Faculdade de Ciências e Tecnologia, Universidade Nova de Lisboa (FCT/UNL), Quinta da Torre, 2829-516 Caparica, Portugal; Centro de Matemática e Aplicações Fundamentais, Universidade de Lisboa (CMAF/UL), 1649-003 Lisboa, Portugal

E-mail address: m1e@fct.un $1 . p t$ 


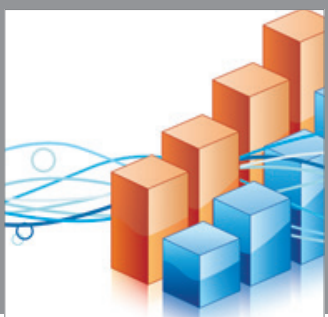

Advances in

Operations Research

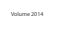

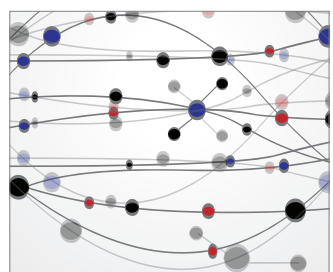

\section{The Scientific} World Journal
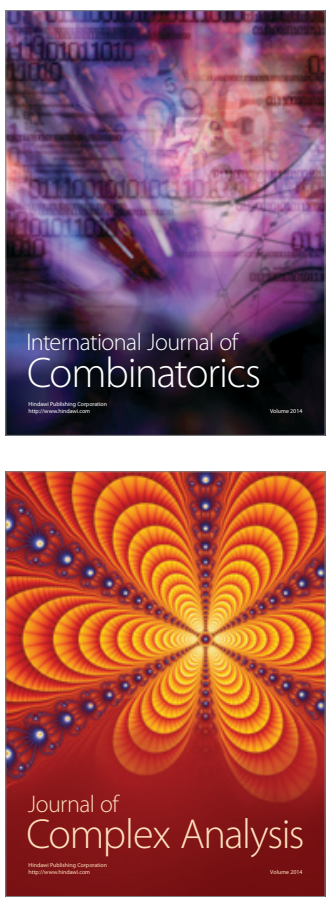

International Journal of

Mathematics and

Mathematical

Sciences
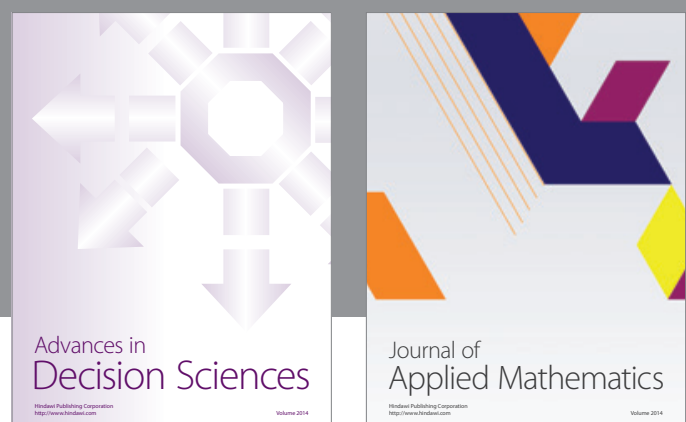

Journal of

Applied Mathematics
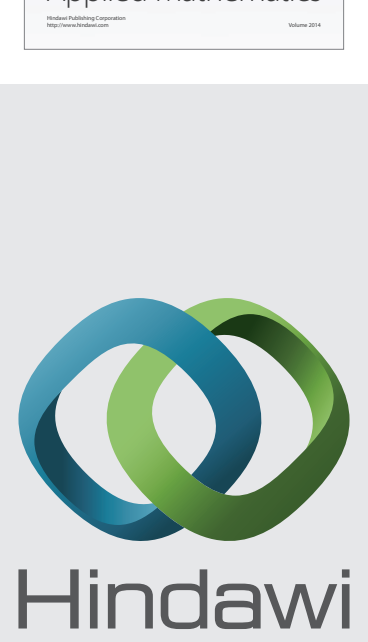

Submit your manuscripts at http://www.hindawi.com
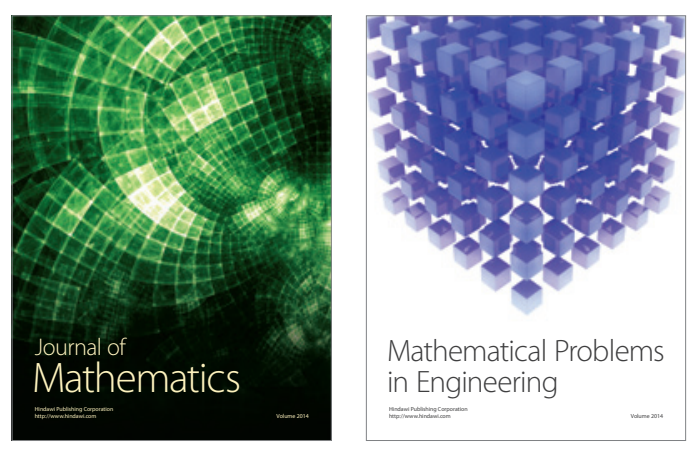

Mathematical Problems in Engineering
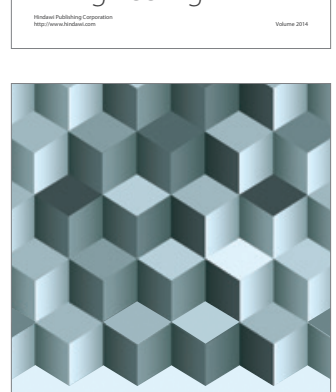

Journal of

Function Spaces
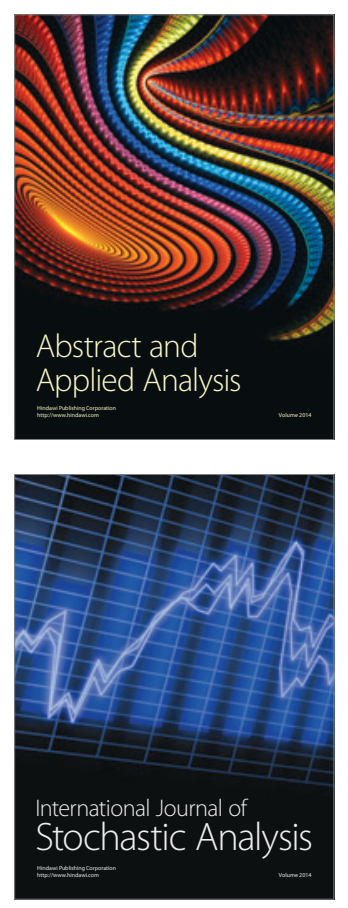

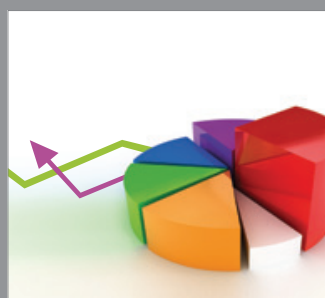

ournal of

Probability and Statistics

Promensencen
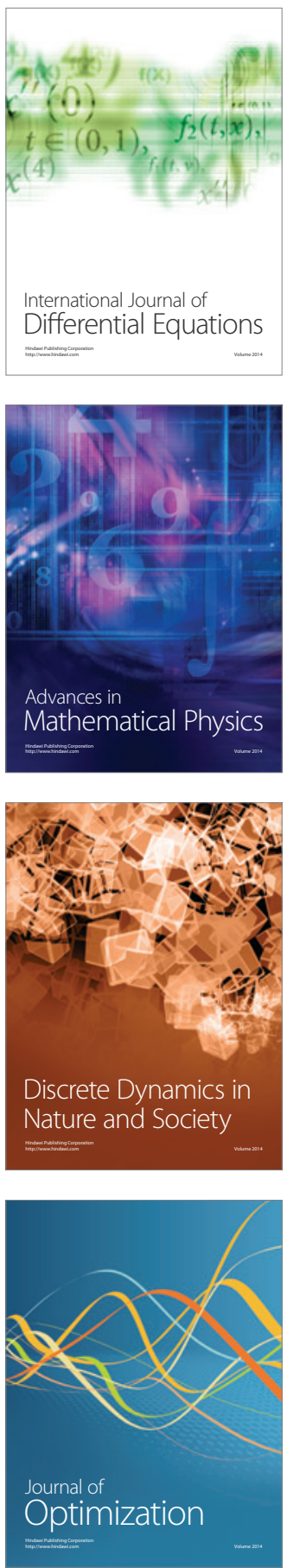